\title{
Using the unified relationship matrix adjusted by breed-wise allele frequencies in genomic evaluation of a multibreed population
}

\author{
M. L. Makgahlela, ${ }^{*}{ }^{1}$ I. Strandén, ${ }^{\star} \dagger$ U. S. Nielsen,‡ M. J. Sillanpää,§\# and E. A. Mäntysaari† \\ *Department of Agricultural Sciences PO Box 27 FIN-00014 University of Helsinki, Finland \\ †MTT Agrifood Research Finland, Biotechnology and Food Research, Biometrical Genetics, FIN-31600 Jokioinen, Finland \\ ‡Danish Agricultural Advisory Service, Udkaersvej 15, 8200 Aarhus, Denmark \\ $\S$ Department of Mathematical Sciences, and \\ \#Department of Biology and Biocenter Oulu, PO Box 3000 FIN-90014 University of Oulu, Finland
}

\begin{abstract}
The observed low accuracy of genomic selection in multibreed and admixed populations results from insufficient linkage disequilibrium between markers and trait loci. Failure to remove variation due to the population structure may also hamper the prediction accuracy. We verified if accounting for breed origin of alleles in the calculation of genomic relationships would improve the prediction accuracy in an admixed population. Individual breed proportions derived from the pedigree were used to estimate breed-wise allele frequencies (AF). Breed-wise and across-breed AF were estimated from the currently genotyped population and also in the base population. Genomic relationship matrices (G) were subsequently calculated using across-breed $\left(\mathbf{G}_{\mathrm{AB}}\right)$ and breed-wise $\left(\mathbf{G}_{\mathrm{BW}}\right)$ AF estimated in the currently genotyped and also in the base population. Unified relationship matrices were derived by combining different $\mathbf{G}$ with pedigree relationships in the evaluation of genomic estimated breeding values (GEBV) for genotyped and ungenotyped animals. The validation reliabilities and inflation of GEBV were assessed by a linear regression of deregressed breeding value (deregressed proofs) on GEBV, weighted by the reliability of deregressed proofs. The regression coefficients $\left(b_{1}\right)$ from $\mathbf{G}_{\mathrm{AB}}$ ranged from 0.76 for milk to 0.90 for protein. Corresponding $b_{1}$ terms from $\mathbf{G}_{\mathbf{B W}}$ ranged from 0.72 to 0.88 . The validation reliabilities across 4 evaluations with different $\mathbf{G}$ were generally 36,40 , and $46 \%$ for milk, protein, and fat, respectively. Unexpectedly, validation reliabilities were generally similar across different evaluations, irrespective of AF used to compute G. Thus, although accounting for the population structure in $\mathbf{G}_{\mathrm{BW}}$ tends to simplify the blending of genomic- and pedigree-based relationships, it appeared to have little effect on the validation reliabilities.
\end{abstract}

Received June 21, 2013.

Accepted October 16, 2013.

${ }^{1}$ Corresponding author: mahlako.makgahlela@helsinki.fi
Key words: allele frequency, adjusted unified relationship matrix, genomic estimated breeding value, admixed cattle population

\section{INTRODUCTION}

Genomic evaluations use genome-wide dense SNP data to predict individual breeding values to be used for selection (Meuwissen et al., 2001). Several reports have shown encouraging results in the application of genomic evaluations within breeds (Hayes et al., 2009b; Su et al., 2010). In dairy cattle, evaluations allow breeders to identify genetically superior bulls at a much earlier age and have been widely applied for breeding purposes (Hayes et al., 2009b; Kearney et al., 2009; Reinhardt et al., 2009; Su et al., 2010). Recent studies have tackled prospects of genomic evaluations in combined purebred (Hayes et al., 2009a; Pryce et al., 2011; Olson et al., 2012) and admixed (Brøndum et al., 2011; Makgahlela et al., 2013a) populations, and have emphasized the potential of this method for multibreed evaluations. The studies concluded that the prediction accuracy across multiple populations was more than that of the parental average but not as effective as the prediction within breeds. The observed low accuracy has been associated with structured reference populations and insufficient linkage disequilibrium (LD) between SNP markers and QTL (de Roos et al., 2009; Ibáñez-Escriche et al., 2009).

Genomic evaluations in dairy cattle are generally implemented in multiple steps (Van Doormaal et al., 2009; VanRaden et al., 2009; Harris and Johnson, 2010; Su et al., 2012b). Each step is characterized by different parameters and assumptions, which may overall compromise the prediction performance. In a single-step approach, the pedigree and genomic information are incorporated into a single relationships matrix, which then enters the mixed-model equations for simultaneous prediction of genomic EBV (GEBV) for genotyped and ungenotyped individuals (Misztal et al., 2009; Christensen and Lund, 2010). In comparison to multi- 
step approaches, this provides a unified framework, minimizes possible errors, and provides the opportunity for more accurate genomic predictions (Aguilar et al., 2010). Although computationally expensive, the prediction accuracies from single-step analyses are generally higher than observed for multi-step procedures (Aguilar et al., 2010; Forni et al., 2011).

A crucial component in single-step analyses is the optimal construction of $\mathbf{G}$, and optimal weighting of the pedigree and genomic information (Forni et al., 2011; Meuwissen et al., 2011; Christensen et al., 2012). Genomic relationships capture unrecorded pedigrees, and are expressed relative to the mean relatedness of the genotyped individuals, because genomic relationship matrices $(\mathbf{G})$ are often calculated using allele frequencies (AF) of the genotyped individuals (VanRaden, 2008; Yang et al., 2010). To be compatible with the pedigree-based relationship matrix $(\mathbf{A}), \mathbf{G}$ is scaled with complex scaling parameters (Chen et al., 2011; Meuwissen et al., 2011; Christensen et al., 2012). In multi-breeds, the construction of $\mathbf{G}$ using AF across breeds, however, tended to increase $\mathbf{G}$ coefficients for animals that were distant from the mean relatedness of the genotyped population (Simeone et al., 2011; Makgahlela et al., 2013b), and closely followed $\mathbf{A}$ when AF within breeds were used to derive $\mathbf{G}$ (Makgahlela et al., $2013 \mathrm{~b}$ ). It was earlier found that $\mathbf{G}$ computed with either AF within or across breeds generated similar validation reliabilities using the genomic BLUP (GBLUP) of genotyped animals only, due to inclusion of the breed mean into the model (Makgahlela et al., 2013b).

We hypothesize that if the accuracy of genomic evaluations depends on the structure of the reference population, then accounting for breed origin of alleles in the calculation of $\mathbf{G}$ in crossbreeds could improve the reliability of GEBV estimated using the unified relationship matrix (single-step GBLUP, ssGBLUP). The Nordic Red dairy cattle (RDC) population has been shown to have a cross-breeding structure (Brøndum et al., 2011; Makgahlela et al., 2013a), low marker-QTL $\mathrm{LD}$, and large effective population size (Rius-Vilarrasa et al., 2011). Therefore, we investigated if accounting for breed composition in $\mathbf{G}$ calculated with currently genotyped AF could improve the reliability of GEBV. Further, we studied if there would be gain in reliability if $\mathbf{G}$ is calculated using estimated base population AF.

\section{MATERIALS AND METHODS}

\section{Phenotype and Genotype Data}

Data were deregressed breeding values (deregressed proofs, DRP) for 2,816,745 cows derived from the EBV using an iterative procedure of Jairath et al. (1998) and
Schaeffer (2001). The cow EBV for milk, protein, and fat and their corresponding effective daughter contributions (EDC) were obtained from March 2010 official Nordic cattle genetic evaluations (http://www.nordicebv.info/Routine+evaluation/). The cow EDC was calculated using ApaX99 software, with an approach described by Interbull (2004) but excluding information provided by the dam. Cows with records had an EDC, indicating the amount of information in the individual animal. Deregression was carried out using the DeRegress option (Strandén and Mäntysaari, 2010) in the MiX99 software package (Lidauer and Strandén, 1999) with full animal model pedigree file. The heritabilities used in deregression were those reported to Interbull (Table 1). In deregression, individual EBV were weighted by their EDC, and the reliability of DRP was as follows: $\mathrm{r}_{\mathrm{DRP}_{i}}^{2}=\mathrm{EDC}_{i} /\left(\mathrm{EDC}_{i}+\lambda\right)$, with $\mathrm{EDC}_{i}$ being EDC for individual $i$ and $\lambda$ being the variance ratio for the trait analyzed. Hereafter, cow DRP and their corresponding EDC contained the original information that would allow the animal model to solve back the original EBV for bulls and cows (Mäntysaari et al., 2011)

Genotype data for bulls were obtained using the Illumina Bovine SNP50 BeadChip (Illumina Inc., 2005). Markers from the X chromosome, without map position in the UMD3.0 genome assembly (Zimin et al., 2009) and with call rate less than $5 \%$ in a large reference sample of Danish Holstein bulls analyzed in the same laboratory were discarded. Further edits removed marker loci with minor allele frequency less than $5 \%$ and animal genotypes with a GenCall score (Illumina Inc., 2005) less than $60 \%$. Finally, we imputed genotypes for missing markers using fastPHASE software (Scheet and Stephens, 2006). After pruning, 4,106 genotyped bulls with 38,194 SNP markers were available for our analyses. These data were divided into a training set of 3,300 bulls and a test set of 806 young bulls. Routine evaluations of the 2005 Nordic cattle genetic evaluations (genetic evaluation is available at

Table 1. The parameters used in the deregression of breeding values (deregressed proofs, DRP): the heritability $\left(\mathrm{h}^{2}\right)$, variance ratio $\left(\lambda=\frac{1-\mathrm{h}^{2}}{\mathrm{~h}^{2}}\right)$, and mean reliability of $\mathrm{DRP}\left(\overline{\mathrm{r}}_{\mathrm{DRP}}^{2}\right)$ for bulls in the training and testing data sets by trait

\begin{tabular}{lcccc}
\hline & & \multicolumn{2}{c}{$\overline{\mathrm{p}}_{\mathrm{DRP}}^{2}$} \\
\cline { 3 - 5 } Trait & $\mathrm{h}^{2}$ & $\lambda$ & $\begin{array}{c}\text { Training } \\
\text { bulls }\end{array}$ & $\begin{array}{c}\text { Testing } \\
\text { bulls }\end{array}$ \\
\hline Milk & 0.40 & 1.50 & 0.96 & 0.95 \\
Protein & 0.28 & 2.57 & 0.95 & 0.93 \\
Fat & 0.32 & 2.13 & 0.95 & 0.94 \\
\hline
\end{tabular}


http://www.nordicebv.info/Routine+evaluation/) were used as a threshold where training bulls were evaluated for the first time before 2005 and validation bulls were not evaluated in 2005.

Breed proportions (BP) for each bull were calculated as shown by Lidauer et al. (2006) using the full pedigree of the RDC, which included 4,624,453 animals. The estimation of BP identified 13 known breeds in this admixed population. Our data included 61 pure base breed individuals and 4,045 animals that were composites of 2 or more base breeds. To choose the breeds for our analyses, we calculated average BP in the data and identified only those main breeds that had average BP over $10 \%$. From this restriction, 4 breeds were defined as Swedish Red, Finnish Ayrshire, Norwegian Red, and the remaining breeds with $\mathrm{BP}$ less than $10 \%$ were combined into a category "other" [see Makgahlela et al. (2013a) for more detailed information of the number of breeds and structure of the RDC population].

\section{The Construction of Relationship Matrices}

The descriptions for the estimation of AF within breed in an admixed population have been given by Makgahlela et al. (2013b). In the currently genotyped population, AF for every marker in each breed were estimated by solving a simple multiple regression of gene content with coding 0,1 , or 2 number of copies of the second allele on breed proportions for the 4 defined breeds. Following the gene content algorithm of Gengler et al. (2007), the regression model above was extended to include the random animal genetic effects in the estimation of AF for ungenotyped ancestral animals. The relationship matrix of animal genetic effects was obtained using the full pedigree of the RDC population. Minimum and maximum thresholds of the estimated $\mathrm{AF}$ within breeds were restricted to 0.05 and 0.95 , respectively, which was equivalent to minor allele frequency less than $5 \%$ requirement. The expectation of $\mathrm{AF}$ for marker $j\left(\hat{\mathrm{P}}_{j}\right)$ from either model is given by $\hat{\mathbf{P}}_{j}=\mathbf{B} \hat{\mathrm{b}}_{j}$, where $\mathbf{B}$ contains individual breed proportions for the Swedish Red, Finnish Ayrshire, Norwegian Red, and combined breeds "other" and their corresponding coefficients $\hat{b}_{j}$ from the current or the base population. Gengler's method was also used to estimate base population AF across breeds.

Approaches for calculating $\mathbf{G}$ within a breed were shown by VanRaden (2008) and Yang et al. (2010). So, for comparison purposes, we also assumed that our admixed population was a single breed and defined $\mathbf{G}$ using across-breed $\mathrm{AF}$ as $\mathbf{G}_{\mathrm{AB}}=\mathbf{Z Z} \mathbf{Z}^{\prime} / \mathrm{k}$, where $\mathbf{Z}$ is a matrix of $n$ genotyped animals and $m$ SNP markers. The coefficients of the $j$ th column in the matrix $\mathbf{Z}$ are $\mathrm{u}_{i j}-2 \mathrm{p}_{j}$, where $\mathrm{u}_{i j}$ has the value 0,1 , or 2 for number of the second allele, $\mathrm{p}_{j}$ is the AF of the second allele at SNP marker $j$ across breeds and $\mathrm{k}=2 \sum_{j} \mathrm{p}_{j}\left(1-\mathrm{p}_{j}\right)$. The estimated breed-wise AF were used to adjust genotypes in the calculation of $\mathbf{G}$ as described in detail by Makgahlela et al. (2013b). The $\mathbf{G}$ adjusted by breedwise $A F$ was defined as $\mathbf{G}_{\mathbf{B W}}=\mathbf{M} \mathbf{M}^{\prime} / m$, where $\mathbf{M}$ was of the same size as matrix $\mathbf{Z}$; however, element $\mathbf{M}_{i j}$ was $\mathrm{u}_{i j}-2 \mathrm{p}_{i j} / \sqrt{2 \mathrm{p}_{i j}\left(1-\mathrm{p}_{i j}\right)}$, where $\mathrm{p}_{i j}$ is the expected AF of the $j$ th marker for individual $i$. Furthermore, $\mathbf{G}_{\mathrm{AB}}$ and $\mathbf{G}_{\mathrm{BW}}$ were also calculated, however, using across-breed and breed-wise AF, respectively, estimated for ungenotyped animals in the base population.

\section{Statistical Analyses}

Optimally, single-step evaluations are based on original data, and evaluation models are the same as in traditional evaluations. However, here our interest was on behavior of the adjusted unified relationship matrix, not the modeling of phenotypic records. The evaluation data excluded daughters of validation bulls, as well as cows born after 2005. Analyses of GEBV for all animals in the pedigree data were performed under the following model:

$$
\mathbf{y}=\mathbf{1}_{\mathrm{n}} \mu+\mathbf{X a}+\mathbf{e}
$$

where $\mathbf{y}$ is a vector of cow DRP for animals with testday records, $\mu$ is the overall mean; $\mathbf{1}_{\mathrm{n}}$ is a unit vector, $\mathbf{a}$ is a vector of additive genetic effects, $\mathbf{X}$ is an incidence matrix relating breeding values to corresponding $\mathrm{DRP}$, and $\mathbf{e}$ is a vector of random residuals. It is assumed that $\mathbf{e} \sim N\left(\mathbf{0}, \mathbf{R} \sigma_{\mathrm{e}}^{2}\right)$, where $\mathbf{0}$ is a vector of zeros, $\sigma_{\mathrm{e}}^{2}$ is the residual variance, and $\mathbf{R}$ is a diagonal matrix with diagonal elements $r_{i i}=1 / w_{i}$, and $w_{i}$ is the number of cow effective record contributions. Genetic effects (a) were assumed to have $\mathrm{E}(\mathbf{a})=0$ and the $\operatorname{Var}(\mathbf{a})=\mathbf{H} \sigma_{\mathrm{a}}^{2}$, where $\sigma_{\mathrm{a}}^{2}$ is the additive genetic variance and $\mathbf{H}$ is a unified relationship matrix that combined genomic $(\mathbf{G})$ - and pedigree (A)-based relationship matrices. As demonstrated in the single-step approach (Aguilar et al., 2010; Christensen and Lund, 2010), the inverse of $\mathbf{H}$ has a simple structure:

$$
\mathbf{H}^{-1}=\mathbf{A}^{-1}+\left[\begin{array}{cc}
\mathbf{G}_{\mathrm{t}}^{-1}-\mathbf{A}_{11}^{-1} & 0 \\
0 & 0
\end{array}\right],
$$

where $\mathbf{A}$ is the pedigree-based numerator relationship matrix for both genotyped and ungenotyped animals, $\mathbf{A}_{11}$ is the submatrix of $\mathbf{A}$ for genotyped animals, and $\mathbf{G}_{t}=(1-t) \mathbf{G}+t \mathbf{A}_{11}$ is the relationship matrix con- 
structed by combining both genomic (i.e., $\mathbf{G}_{\mathrm{AB}}$ or $\mathbf{G}_{\mathrm{BW}}$ ) and pedigree information. To make $\mathbf{G}_{\mathrm{AB}}$ compatible in scale with $\mathbf{A}_{11}$, a correction factor $r=\frac{\operatorname{trace}\left(\mathbf{A}_{11}\right)}{\operatorname{trace}(\mathbf{G})}$ was used to scale $\mathbf{G}_{\mathbf{A B}}$ before $\mathbf{G}_{\mathbf{A B}}$ and $\mathbf{A}_{11}$ were combined. This scaling was not necessary for $\mathbf{G}_{\mathrm{BW}}$ because the use of breed-wise AF was expected to adjust this matrix to the same level as A. Also, genomic evaluations converged similarly with or without the scaling factor. The constant $t$ defined the weight on pedigree relationships $\mathbf{A}_{11}$. Weights tested by Aguilar et al. (2010) were in the range 0.02 to 0.10 , and had only small effect on predictions. We carried out preliminary analyses by varying the value of $t$ between 0.10 and 0.25 to find the optimal weight for this population. The highest prediction accuracy was achieved when $t$ was 0.20 . Similar weight on $\mathbf{A}$ is currently used in multi-step routine evaluations in Canada (Van Doormaal et al., 2009) and the United States (Sullivan and VanRaden, 2009).

Following the Interbull GEBV validation procedure (Mäntysaari et al., 2010), the validation reliability of GEBV for bulls in the test set was assessed as

$$
\mathbf{y}=1 \mathrm{~b}_{0}+\mathrm{b}_{1} \hat{\mathbf{a}}+\mathbf{e},
$$

where $\mathbf{y}$ is a vector of DRP of test bulls, derived from 2010 routine evaluations and $\hat{\mathbf{a}}$ is a vector of GEBV for these bulls. The linear model was weighted by the reliability of bull DRP, calculated as $\mathrm{r}_{\mathrm{DRP}}^{2}=\mathrm{EDC}_{i} /\left(\mathrm{EDC}_{i}+\lambda\right)$, where $\mathrm{EDC}_{i}$ is the number of effective daughter contributions included in the DRP, $\lambda$ $=\left(4-\mathrm{h}^{2}\right) / \mathrm{h}^{2}$, and heritabilities as presented in Table 1 are derived from traditional evaluations. The validation reliability was defined as $\mathrm{R}_{\mathrm{GEBV}}^{2}=\frac{\mathrm{r}_{(\mathrm{DRP}, \mathrm{GEBV})}^{2}}{\overline{\mathrm{r}}_{\mathrm{DRP}}^{2}}$, where $\overline{\mathrm{r}}_{\mathrm{DRP}}^{2}$ are the average reliabilities of testing bull DRP (Table 1 ). Here, coefficients $b_{0}$ and $b_{1}$ were used to assess the bias in the model.

\section{RESULTS AND DISCUSSION}

The objective of this study was to assess the effect of accounting for the population structure in the calculation of $\mathbf{G}$ on the validation reliability of GEBV from ssGBLUP in the admixed RDC population. The calculations of $\mathbf{G}_{\mathrm{AB}}$ with method 1 or 2 of VanRaden (2008) were found to produce similar relationship estimates (Makgahlela et al., 2013b). The current ssGBLUP was also tested using both methods, with marginal differences in validation reliabilities. We present $\mathbf{G}_{\mathrm{AB}}$ from method 1 due to its extensive use in practical evaluations. Table 2 presents standard deviations of the EBV and GEBV for bulls and daughters of bulls in the training and testing set. Standard deviations were derived within birth year and pooled across years to account for selection. In the training set, standard deviations of the bull GEBV follow very close standard deviations of the standardized pedigree-based EBV, although overall, little variation existed in the standard deviations of bulls and bull daughters among genomic evaluation methods. In the testing set, the bull standard deviation of GEBV is clearly higher than that of the EBV, due to added information from genotypes. The increase appeared higher when the genomic relationships were based on base population AF. Genomic information for test bulls also increased the standard deviation of the GEBV of their daughters. In both the training and the testing sets, the standard deviation of GEBV was higher for bulls than bull daughters, which is expected and due to higher accuracy and genetic progress in bulls.

Table 3 shows the intercepts $\left(b_{0}\right)$, regression coefficients $\left(b_{1}\right)$, and validation reliabilities $\left(\mathrm{R}_{\mathrm{GEBV}}^{2}\right)$ from ssGBLUP with $\mathbf{G}_{\mathrm{AB}}$ and $\mathbf{G}_{\mathrm{BW}}$. Coefficients and $\mathrm{R}_{\mathrm{GEBV}}^{2}$ values are shown when these matrices were constructed with the observed and base population AF. The values of intercepts were about the same for all traits because the original EBV indices were standardized to have standard deviation of 10 . The average DRP of bulls in the training data were $90.32,86.18$, and 89.89 for milk, protein, and fat, respectively. If the validation $b_{1}$ values were equal to 1 , the expected intercepts from the regression analyses should be close to these values. However, regression coefficients for all traits were less than 1 .

Coefficients of regression ranged from 0.78 to 0.90 for protein, from 0.80 to 0.85 for fat, and from 0.72 to 0.77 for milk (Table 3 ). The $b_{1}$ terms for all traits were generally higher when $\mathbf{G}_{\mathrm{AB}}$ and $\mathbf{G}_{\mathrm{BW}}$ were computed using observed AF. With $\mathbf{G}_{\mathrm{BW}}$ and base population $\mathrm{AF}, \mathrm{b}_{1}$ were smaller by $0.03,0.10$, and 0.04 units for milk, protein, and fat, respectively. With $\mathbf{G}_{\mathrm{AB}}$, the corresponding $b_{1}$ terms were smaller relative to observed $\mathrm{AF}$ by $0.01,0.04$, and 0.03 units, respectively. In all the traits, when accounting for breed composition in $\mathbf{G}_{\mathrm{BW}}$, the regression coefficients were smaller than when assuming the same population in $\mathbf{G}_{\mathrm{AB}}$. The differences in $b_{1}$ values between $\mathbf{G}_{\mathrm{AB}}$ and $\mathbf{G}_{\mathrm{BW}}$ were 2 units for milk and protein using observed $\mathrm{AF}$ but increased to 4 and 8 units for these traits when $\mathbf{G}_{\mathrm{AB}}$ and $\mathbf{G}_{\mathrm{BW}}$ were constructed with base population AF. Thus, using base population AF resulted in more inflated GEBV than observed AF. According to Interbull protocol for GEBV validation, the expected value for $b_{1}$ from the validation model is 1 , on the condition that no preselection exists based on EBV or DRP when genotyping animals (Mäntysaari et al., 2010). For all methods and 
Table 2. Standard deviations of $\mathrm{EBV}^{1}$ (pedigree-based relationship matrix, Ped) and genomic EBV (GEBV) in the training and testing data and for bull daughters in the training and testing data ${ }^{2}$

\begin{tabular}{|c|c|c|c|c|c|c|c|}
\hline \multirow[b]{2}{*}{ Data $^{3}$} & \multirow[b]{2}{*}{ Method $^{4}$} & \multicolumn{2}{|c|}{ Milk } & \multicolumn{2}{|c|}{ Protein } & \multicolumn{2}{|c|}{ Fat } \\
\hline & & Bulls & Daughters & Bulls & Daughters & Bulls & Daughters \\
\hline Training data & Ped & 9.08 & 8.69 & 9.06 & 7.99 & 9.43 & 8.18 \\
\hline \multirow[t]{2}{*}{ Observed AF } & $\mathrm{G}_{\mathrm{AB}}$ & 9.06 & 8.69 & 9.04 & 7.99 & 9.42 & 8.17 \\
\hline & $\mathrm{G}_{\mathrm{BW}}$ & 9.09 & 8.69 & 9.08 & 7.99 & 9.46 & 8.18 \\
\hline \multirow{2}{*}{ Base AF } & $\mathrm{G}_{\mathrm{AB}}$ & 9.07 & 8.69 & 9.05 & 7.99 & 9.43 & 8.17 \\
\hline & $\mathrm{G}_{\mathrm{BW}}$ & 9.07 & 8.69 & 9.07 & 7.99 & 9.44 & 8.18 \\
\hline Testing data & Ped & 6.02 & 4.83 & 5.29 & 4.32 & 5.82 & 4.71 \\
\hline \multirow[t]{2}{*}{ Observed AF } & $\mathrm{G}_{\mathrm{AB}}$ & 6.86 & 5.11 & 6.56 & 4.73 & 7.04 & 5.08 \\
\hline & $\mathrm{G}_{\mathrm{BW}}$ & 7.02 & 5.20 & 6.67 & 4.78 & 7.10 & 5.12 \\
\hline \multirow[t]{2}{*}{ Base AF } & $\mathrm{G}_{\mathrm{AB}}$ & 7.02 & 5.17 & 6.87 & 4.82 & 7.26 & 5.16 \\
\hline & $\mathrm{G}_{\mathrm{BW}}^{\mathrm{AD}}$ & 7.33 & 5.31 & 7.43 & 5.07 & 7.40 & 5.23 \\
\hline
\end{tabular}

${ }^{1}$ Estimated breeding values are expressed as standardized breeding values with SD of 10 units for bulls born between the years 2002 and 2004.

${ }^{2}$ Standard deviations were calculated within birth year and pooled across years.

${ }^{3} \mathrm{AF}=$ allele frequencies.

${ }^{4} \mathbf{G}_{\mathrm{AB}}=$ genomic relationship matrix derived using original method 1 of VanRaden (2008), with across-breed $\mathrm{AF} ; \mathbf{G}_{\mathrm{BW}}=$ genomic relationship matrix derived following method 2 of VanRaden (2008), with breed-wise AF.

traits in the current study, the $\mathrm{b}_{1}$ values were far less than the expected value of 1 , which means that GEBV were inflated, resulting in overprediction of DRP. The genotype data included all proven bulls in candidate birth-year classes, which rules out a possibility for selective genotyping among animals. On the other hand, the GEBV seemed more inflated (regression coefficients decreased) when accounting for breed origin of alleles than assuming that this admixed population is single and uniform, and furthermore, when $\mathbf{G}_{\mathrm{AB}}$ and $\mathbf{G}_{\mathrm{BW}}$ were constructed with base population AF.

The range of regression coefficients observed from $\mathbf{G}_{\mathrm{BW}}$ were consistent with the literature for single-step evaluations in single populations (Aguilar et al., 2010; Vitezica et al., 2011; Gao et al., 2012) and when assuming single population for the RDC (Koivula et al., 2012; $\mathrm{Su}$ et al., 2012b). Similar values were also found for milk and protein when accounting for population structure in single-step (Harris et al., 2012) and multi-step (Makgahlela et al., 2013a) evaluations. The inflation of GEBV has been a subject of great concern for genomic evaluations and is generally associated with suboptimal blending of information from genotyped and ungenotyped but phenotyped individuals (Meuwissen et al., 2011; Olson et al., 2011; Vitezica et al., 2011). In particular, bias in single-step evaluations has been linked with imprecise derivation of G (Aguilar et al., 2010; Chen et al., 2011; Forni et al., 2011; Meuwissen et al., 2011) and suboptimal incorporation of pedigree-based and marker-based relationships (Meuwissen et al., 2011; Vitezica et al., 2011; Christensen et al., 2012). The main cause remains uncertain; however, previous reports have suggested that the observed bias could be reduced by scaling $\mathbf{G}$ (Forni et al., 2011), adding a

Table 3. Intercepts $\left(\mathrm{b}_{0}\right)$, regression coefficients $\left(\mathrm{b}_{1}\right)$, and validation reliabilities $\left(\mathrm{R}_{\mathrm{BV}}^{2}\right)$ of genomic and pedigreebased breeding values (pedigree-based relationship matrix, Ped) for testing bulls by trait, estimated using different adjusted unified relationship matrices

\begin{tabular}{|c|c|c|c|c|c|c|c|c|c|}
\hline \multirow[b]{2}{*}{ Method $^{1}$} & \multicolumn{3}{|c|}{ Milk } & \multicolumn{3}{|c|}{ Protein } & \multicolumn{3}{|c|}{ Fat } \\
\hline & $\mathrm{b}_{0}$ & $\mathrm{~b}_{1}$ & $\mathrm{R}_{\mathrm{BV}}^{2}$ & $\mathrm{~b}_{0}$ & $\mathrm{~b}_{1}$ & $\mathrm{R}_{\mathrm{BV}}^{2}$ & $\mathrm{~b}_{0}$ & $\mathrm{~b}_{1}$ & $\mathrm{R}_{\mathrm{BV}}^{2}$ \\
\hline $\begin{array}{l}\text { Ped } \\
\text { Observed AF }\end{array}$ & 81.84 & 0.72 & 0.24 & 70.64 & 0.89 & 0.25 & 79.56 & 0.81 & 0.28 \\
\hline $\mathrm{G}_{\mathrm{AB}}$ & 84.06 & 0.77 & 0.37 & 76.11 & 0.90 & 0.40 & 82.81 & 0.85 & 0.47 \\
\hline $\begin{array}{c}\mathbf{G}_{\mathrm{BW}} \\
\text { Base AF }\end{array}$ & 84.83 & 0.75 & 0.36 & 77.49 & 0.88 & 0.39 & 83.43 & 0.84 & 0.47 \\
\hline $\mathbf{G}_{\mathrm{AB}}$ & 84.36 & 0.76 & 0.37 & 76.90 & 0.86 & 0.40 & 83.58 & 0.82 & 0.47 \\
\hline $\mathrm{G}_{\mathrm{BW}}$ & 85.39 & 0.72 & 0.36 & 79.69 & 0.78 & 0.38 & 84.18 & 0.80 & 0.46 \\
\hline
\end{tabular}


small value to all elements of this matrix (Chen et al., 2011), or doing both (Christensen et al., 2012). The purpose here is to get relationships in $\mathbf{G}$ to be defined with respect to the same base generation as in $\mathbf{A}$. To address these issues differently, we defined $\mathbf{G}_{\mathrm{BW}}$ with respect to individuals' breed origin and examined its ability when the base generation was defined in the current or distant past generation, without further need for complex scaling, but found no improvement on the inflation of GEBV.

The validation reliabilities for fat were all similar and about $47 \%$ across the 4 different evaluations (Table 3 ). However, reliability levels for milk and protein were $1 \%$ lower for $\mathbf{G}_{\mathrm{BW}}$ calculated with observed $\mathrm{AF}$ and $2 \%$ lower for protein when $\mathbf{G}_{\mathbf{B W}}$ was derived with base population AF. The validation reliabilities across methods were overall slightly higher for protein and fat (about $40 \%$ ) and lower for milk ( $\sim 36 \%$ ). These levels of reliability were unexpected, and indicate that accounting for breed composition in $\mathbf{G}_{\mathrm{BW}}$ appeared to have less effect on the validation reliabilities, even when evaluation models included genotyped and ungenotyped individuals. The predictions from $\mathbf{G}_{\mathrm{BW}}$ generally resulted in $1 \%$ less reliable GEBV than $\mathbf{G}_{\mathrm{AB}}$, regardless of $\mathrm{AF}$ type. Pedigree-based EBV reliabilities were lower than GEBV by at least $12 \%$ for milk and protein and $18 \%$ for fat. It is not straightforward to compare our results with existing reports, mainly because of differences in population structures, statistical methods, and phenotypes. Harris et al. (2012), which was the only study that looked into the prospect of using $\mathbf{G}$ adjusted for breed composition in single-step evaluation of GEBV, also found only small differences between validation reliabilities in a multibreed population of purebred Holsteins and Jerseys and their crossbred individuals. Apart from a different population structure to ours, those authors used test-day records for similar traits as phenotypes in their evaluations and found similar accuracies, especially when validation was done separately for crossbred animals.

Using the US Holstein cattle population, Aguilar et al. (2010) compared $R_{\text {GEBV }}^{2}$ values for final score from ssGBLUP when $\mathbf{G}$ matrices were computed with AF from the current population, base population, or using 0.5 for all markers. The validation reliabilities using EBV or daughter deviations as data were $4 \%$ higher with $\mathrm{AF}$ of 0.5 than using current or base population $\mathrm{AF}$, and were similar between $\mathbf{G}$ matrices computed with AF from the current or base population. The AF of 0.5 equalizes the variance contribution of homozygous alleles 1 and 2 to the diagonal elements of $\mathbf{G}$, whereas unequal $\mathrm{AF}$ in the calculation of $\mathbf{G}$ tends to give more weight to rare alleles. In contrast, Chen et al.
(2011) used single-step evaluations in pure broiler chicken lines and observed that prediction accuracies with $\mathbf{G}$ calculated with observed AF were generally 1 to $2 \%$ higher than equal $\mathrm{AF}$ for all markers. This was somewhat in agreement with slightly higher reliabilities we found when using observed AF to build G. The predictive ability in their study was defined as the correlation between the genomic value and phenotype (i.e., BW and breast meat). Forni et al. (2011), in the evaluation of pig litter size, found correlations close to 1.0 between GEBV derived from $\mathbf{G}$ calculated with either observed AF, AF equal 0.5 for all markers, or normalized to have average diagonals equal to 1 . Such equally good prediction ability has also been observed in multistep GBLUP evaluations in single- (VanRaden, 2008) and multi-breed (Makgahlela et al., 2013b) populations. In the latter study, it was thought that predictions with $\mathbf{G}_{\mathrm{BW}}$ may have suffered from using GBLUP for genotyped animals only, as models included breed proportions as fixed regressions and this would transfer breed means back into genomic values as suggested by Strandén and Christensen (2011). Thus, Makgahlela et al. (2013b) suggested that accounting for breed AF would be beneficial only in single-step evaluation of genotyped and ungenotyped individuals. This advantage was, however, not achieved with ssGBLUP in the current study. The correlations between GEBV from different G were all equal to 1 for animals in the test data. As shown in Table 4 and in agreement with Forni et al. (2011), these correlations were close to 1 when using observed AF for validation animals. However, correlations with $\mathbf{G}_{\mathrm{BW}}$ decreased slightly when this matrix was derived with base population AF.

More closely, we examined the differences between GEBV from $\mathbf{G}_{\mathrm{AB}}$ and $\mathbf{G}_{\mathrm{BW}}$ to see if any patterns existed between methods for testing individuals in different populations and also to check if predictions were in accordance, as it was earlier observed that the calculation of $\mathbf{G}_{\mathrm{AB}}$ behaved differently in different subpopulations (Makgahlela et al., 2013b). This is because although the RDC constitutes populations from Sweden (SWE), Finland (FIN), and Denmark (DNK), the latter is genetically more distant from the first 2 , thereby decreasing the extent of LD (Rius-Vilarrasa et al., 2011) and validation reliabilities (Brøndum et al., 2011). In Figure 1, the difference in GEBV estimates for candidate bulls obtained from the model with $\mathbf{G}_{\mathrm{AB}}$ and that with $\mathbf{G}_{\mathrm{BW}}$ are plotted against the DRP of the bull. As shown in the figure, very small differences existed between estimates when the observed AF were used to calculate these matrices. However, when the base population AF were used, the differences between methods were greater than 3 standard deviations for some individu- 
Table 4. Correlations between genomic breeding values from different genomic relationship matrices in the testing data set by trait

\begin{tabular}{|c|c|c|c|c|c|}
\hline Trait & Method $^{1}$ & Allele frequency & $\mathrm{G}_{\mathrm{BW}}$ & $\mathrm{G}_{\mathrm{AB}}{ }^{2}$ & $\mathrm{G}_{\mathrm{BW}}{ }^{2}$ \\
\hline \multirow[t]{3}{*}{ Milk } & $\mathrm{G}_{\mathrm{AB}}$ & Population & \multirow[t]{3}{*}{0.99} & 0.99 & 0.97 \\
\hline & $\mathrm{G}_{\mathrm{BW}}$ & Population & & 0.99 & 0.98 \\
\hline & $\mathrm{G}_{\mathrm{AB}}$ & Base & & & 0.99 \\
\hline \multirow[t]{3}{*}{ Protein } & $\mathrm{G}_{\mathrm{AB}}$ & Population & \multirow[t]{3}{*}{0.99} & 0.99 & 0.95 \\
\hline & $\mathrm{G}_{\mathrm{BW}}$ & Population & & 0.99 & 0.97 \\
\hline & $\mathrm{G}_{\mathrm{AB}}$ & Base & & & 0.98 \\
\hline \multirow[t]{3}{*}{ Fat } & $\mathrm{G}_{\mathrm{AB}}$ & Population & \multirow[t]{3}{*}{0.99} & 0.99 & 0.98 \\
\hline & $\mathrm{G}_{\mathrm{BW}}$ & Population & & 0.99 & 0.99 \\
\hline & $\mathrm{G}_{\mathrm{AB}}$ & Base & & & 0.99 \\
\hline
\end{tabular}

${ }^{1} \mathbf{G}_{\mathrm{AB}}=$ genomic relationship matrix derived using original method 1 of VanRaden (2008) calculated using across-breed allele frequencies; $\mathbf{G}_{\mathrm{BW}}=$ genomic relationship matrix derived using adjusted method 2 of VanRaden (2008) calculated using breed-wise allele frequencies.

${ }^{2}$ Allele frequency from the base population.

als. These differences were more evident for milk and protein than for fat, which could be dependent on the genetic architecture of the trait. The animals with great differences between methods were generally registered in DNK. This means that evaluations from $\mathbf{G}_{\mathrm{BW}}$ and $\mathbf{G}_{\mathrm{AB}}$ derived with base population AF were similar for animals born SWE and FIN but different for about 15\% of animals born in DNK. Upon further inspection, we found that these individuals had less pedigree information content. They had the highest diagonal elements in $\mathbf{G}_{\mathrm{AB}}$, and although reduced by accounting for $\mathrm{AF}$ within breeds, their diagonal elements were still higher than other animals in $\mathbf{G}_{\mathbf{B W}}$ (Makgahlela et al., 2013b). This could be due to developed methods for the calculation of $\mathbf{G}$, which tends to increase diagonal elements for individuals that are genetically less related to the rest of the genotyped individuals in the populations (Makgahlela et al., 2013b), thereby inflating their genomic values. We found no particular association between the differences between methods and the bull EDC. Scatter plots of DRP against GEBV from analyses with $\mathbf{G}_{\mathrm{BW}}$ and $\mathbf{G}_{\mathrm{AB}}$ were very similar for all traits. Also, similar patterns were found between $\mathbf{G}_{\mathrm{BW}}$ and $\mathbf{G}_{\mathrm{AB}}$ when their predicted values, diagonal elements, or inverses of their diagonal elements were plotted against residuals from the validation model (results not shown).

Genomic selection was proposed for livestock breeding for over a decade ago (Meuwissen et al., 2001). The application of this method has been successful so far for single uniform populations, especially the Holstein dairy cattle populations (Hayes et al., 2009b; Sullivan and VanRaden, 2009; Van Doormaal et al., 2009; Su et al., 2010). However, genomic evaluations have not been as successful for small and admixed populations due to insufficient training data (Thomasen et al., 2012), very low marker-QTL LD (Daetwyler et al., 2008; Brøndum et al., 2011; Pryce et al., 2011; Rius-Vilarrasa et al., 2011), and large effective population sizes (Rius-
Vilarrasa et al., 2011). Although the observed accuracy in admixed populations is greater than that of the parental average, it is low for successful marketing of genomically tested bulls. For effective use in breeding programs, predictions would need to be unbiased and reliable.

To our knowledge, no underlying genetic mechanism has been explicitly singled out as a cause for less predictive power in admixed populations. In genome-wide association studies, spurious associations and the inflation problems have been reviewed (Price et al., 2010), with similar outcomes as ours when accounting for the population structure (Sul and Eskin, 2013). With plant data, similar outcomes between genomic evaluation models with and without population correction factors were reported in a population known to show considerable substructure (Janss et al., 2012).

The main challenge, though, is that these evaluations rely on the phase of the marker-QTL LD being similar in the training data, where SNP effects are derived, and in the test data. Thus, in multibreeds, genetic homogeneity should exist and the marker-QTL LD should be similar across populations for marker effects to be equal in different populations. As the genetic relationships between populations diverge, genetic homogeneity between populations, however, becomes less likely, due to factors such as genotype-by-environment and genotypeby-genetic background interactions (de Roos et al., 2009). Given this, de Roos et al. (2009) highlighted a need for much denser marker data, to capture LD that persists across populations. Still, only 2 to $3 \%$ gain was achieved using approximately 800,000-marker (800K) density chips with real data (Harris et al., 2011; Su et al., 2012a). Furthermore, small gains were reported when fitting models with breed-specific effects of markers for multibreeds (Ibáñez-Escriche et al., 2009; Olson et al., 2012; Makgahlela et al., 2013a). All studies were conducted in different populations or simulating dif- 
Observed population AF
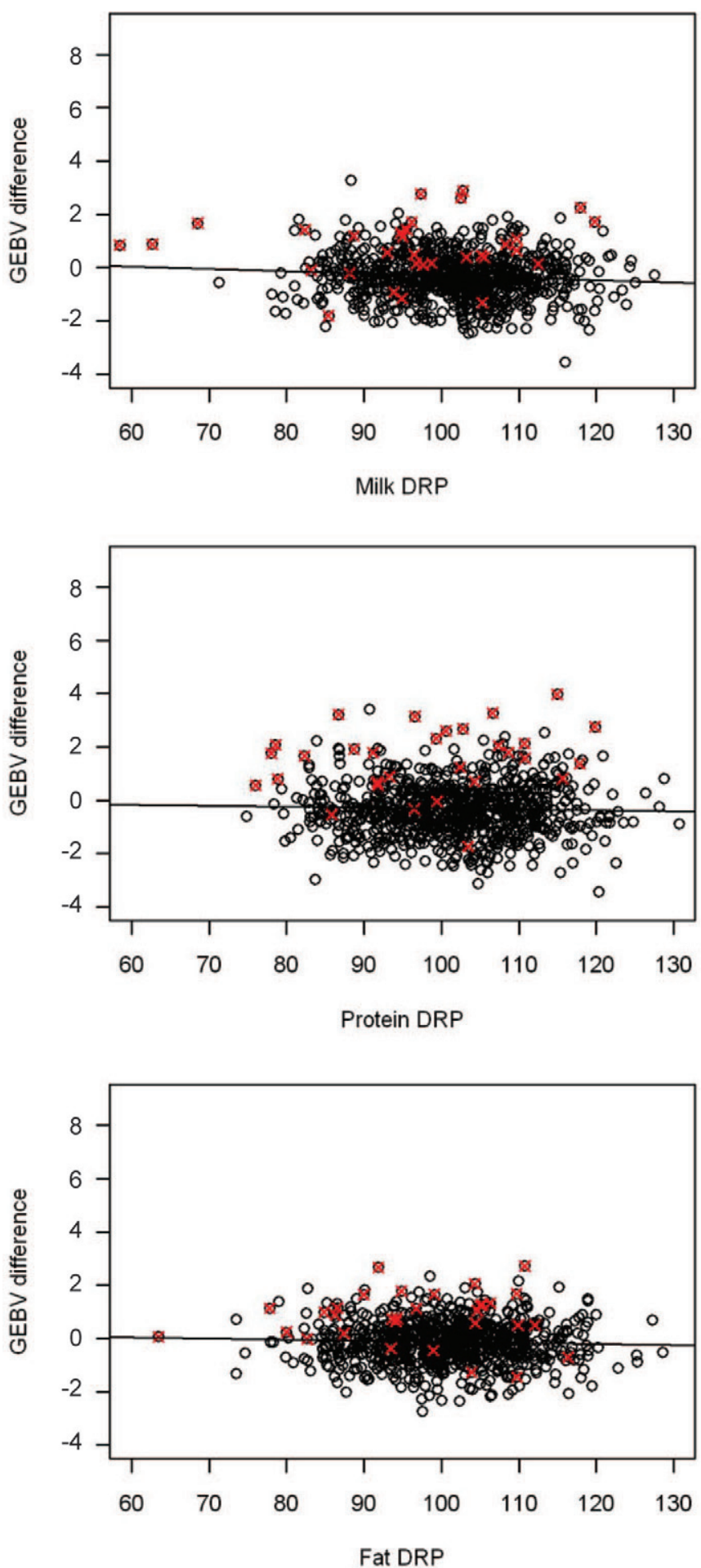

Base population AF
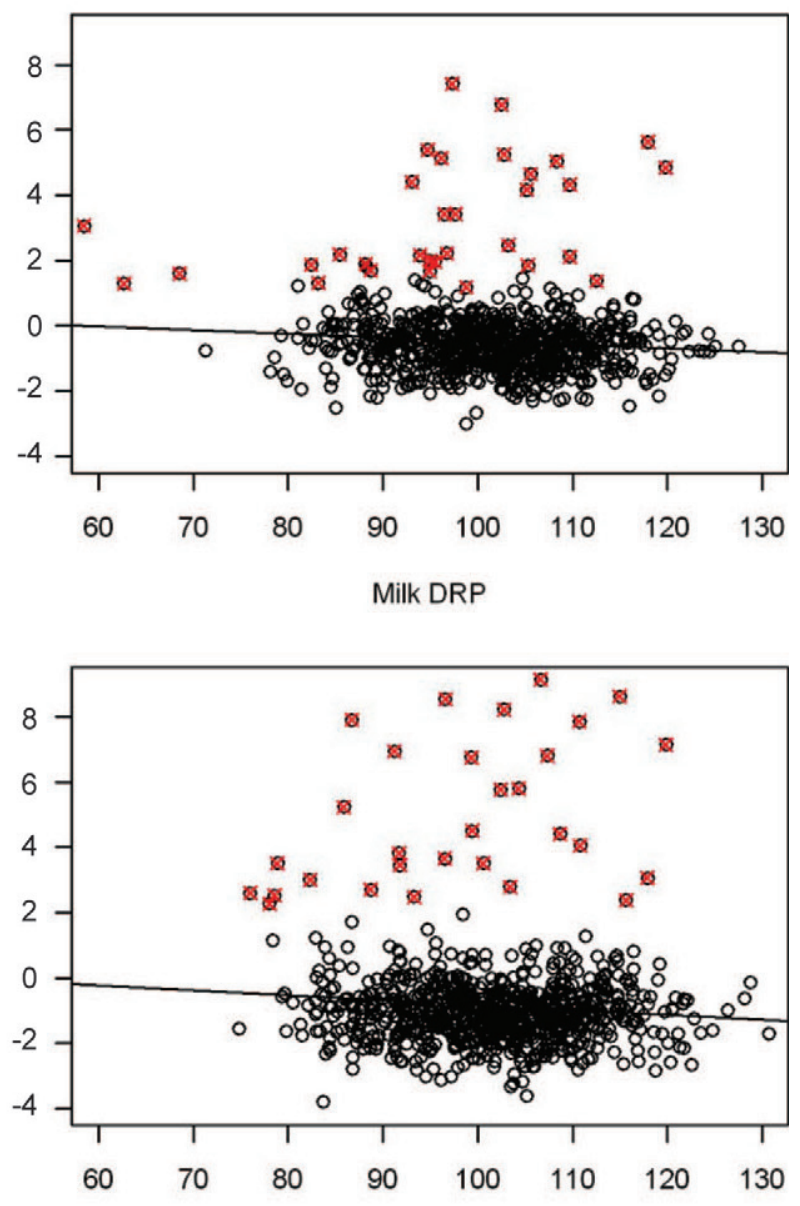

Protein DRP

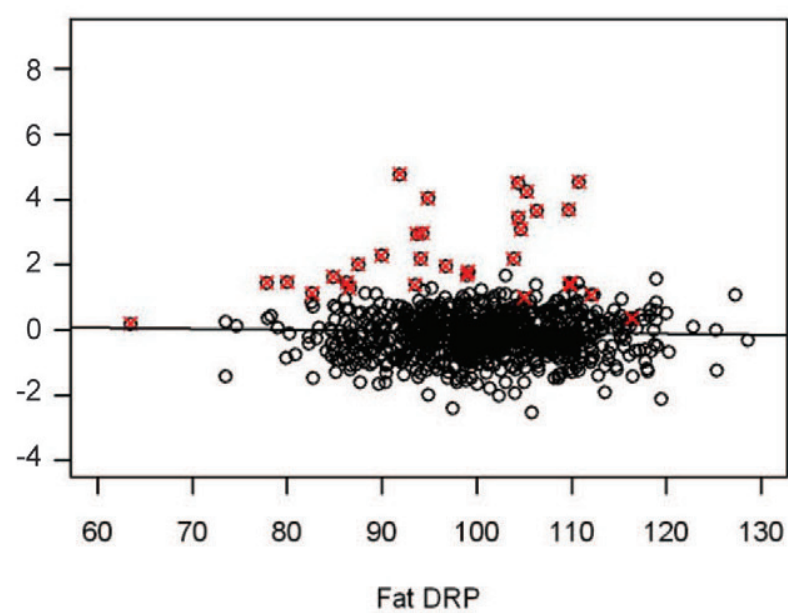

Figure 1. Scatter plots of the difference between genomic EBV (GEBV) from original method $1\left(\mathbf{G}_{\mathrm{AB}}\right)$ and adjusted method $2\left(\mathbf{G}_{\mathrm{BW}}\right)$ of VanRaden (2008) versus deregressed breeding values (deregressed proofs, DRP) by trait. $\mathbf{G}_{\mathbf{A B}}=$ genomic relationship matrix derived using original method 1 of VanRaden (2008), calculated using across-breed allele frequencies; $\mathbf{G}_{\mathrm{BW}}=$ genomic relationship matrix derived using adjusted method 2 of VanRaden (2008), calculated using breed-wise allele frequencies (AF). GEBV difference $=$ the difference between GEBV from $\mathbf{G}_{\mathrm{AB}}$ and $\mathbf{G}_{\mathrm{BW}}$. Data points with $\times$ show patterns of individuals that tended to differ between methods. Color version available in the online PDF. 
ferent admixture levels and the consensus is that the increase in prediction accuracy is generally negligible. This may confirm the suggestion of Deng (2001) that, although the effect of LD has been well documented, population admixture per se also has the potential to mask or induce the genetic effects of QTL underlying quantitative traits, which reduces the predictive power of models.

The genetic structure of the RDC is very unique, with over $95 \%$ of individuals being composites of base breeds. This population has been subjected to the same breeding goal for over 2 decades, which is expected to maintain the average AF and unify marker-QTL LD across populations. We considered the possibility of estimating marker-based breed proportions as opposed to expected pedigree-based breed proportions. However, lack of pure base breed animals constrained this effort because the proposed methods generally require the presence of these individuals for the estimation of breed-specific AF (Kuehn et al., 2011; Frkonja et al., 2012). In using breed proportions to define base breeds when accounting for breed origin of alleles in $\mathbf{G}$, it was expected that the $\mathbf{G}_{\mathrm{BW}}$ would yield unbiased and more accurate predictions. This is because it reduces the complexity in the calculation of the unified matrix $\mathbf{H}^{-1}$ in 2 ways. First, $\mathbf{G}_{\mathrm{BW}}$ redefines the expected relationships across and within DNK, SWE, and FIN populations, as they would likely appear when using pedigree information (Makgahlela et al., 2013b). Second, no need existed for further complex scaling parameters (Aguilar et al., 2010; Gao et al., 2012) in $\mathbf{G}_{\mathrm{BW}}$, as the adjustment appeared to make it more compatible with A. This would have been especially evident when this matrix was derived with base population $\mathrm{AF}$, as it was earlier found that its diagonal elements were more correlated with $\mathbf{A}$ than those from $\mathbf{G}_{\mathbf{A B}}$ (Makgahlela et al., 2013b). However, genomic predictions from $\mathbf{G}_{\mathrm{AB}}$ and $\mathbf{G}_{\mathrm{BW}}$ from all 4 evaluations converged to similar solutions. Although this was the case, the interesting outcome was that after accounting for AF within breeds in $\mathbf{G}_{\mathrm{BW}}$, our model did not require any scaling to make it compatible with $\mathbf{A}$, but achieved similar prediction accuracy as found for $\mathbf{G}_{\mathrm{AB}}$, which uses complex scaling parameters with strong assumptions. Thus, blending of genomic and pedigree information may have been optimized. Also, it should be emphasized that the method used to derive $\mathbf{G}_{\mathrm{BW}}$ was better than $\mathbf{G}_{\mathrm{AB}}$, as the latter was found to have distorted diagonal elements (Makgahlela et al., 2013b).

In the presence of suitable data with distinct base breeds, it may be useful to calculate breed proportions using SNP marker data instead of pedigree. This information has been shown to be very accurate in relation to pedigree estimates (Kuehn et al., 2011; Frkonja et al., 2012) but the added advantage from markers would come from having actual local ancestry at typed loci. On the other hand, although genomic selection was originally proposed using marker haplotypes instead of single markers (Meuwissen et al., 2001), most studies in our discussion only focused their predictions using individual SNP. A haplotype block constitutes a sequence of neighboring markers on a chromosome. As already mentioned, evaluations work successfully, provided that marker haplotypes or markers are in sufficient LD with the QTL. Haplotype models have been shown to perform better than single markers, especially with low marker density and marker-QTL LD (Calus et al., 2008; de Roos et al., 2011). As more markers are included into a haplotype, a haplotype block becomes distinct; the probability of marker haplotypes being associated by chance is reduced, thereby increasing the probability of originating from a common ancestry. Thus, haplotypes will improve the specificity among patterns of marker-QTL LD, as haplotypes will be associated with unique QTL alleles, which will increase the QTL variance explained by the haplotype (Calus et al., 2008). Because the observed LD is low in multibreed and admixed populations, which results in low predictive power, haplotype models may be more advantageous over estimating individual SNP marker effects.

\section{CONCLUSIONS}

We compared the validation reliabilities of GEBV from ssGBLUP using genomic relationship matrices constructed ignoring structure with AF across breed, and $\mathbf{G}$ built, accounting for breed composition with estimated breed-wise AF, in the admixed RDC population. Both matrices were constructed using AF from either the observed genotypes or from the base population. In general, the evaluations from both matrices gave about the same reliabilities. Thus, genomic predictions, when accounting for breed origin of alleles, resulted in similar validation biases and reliabilities as assuming that this admixed population had a single uniform structure. In both cases, the use of observed AF in the calculation of $\mathbf{G}$ resulted in slightly higher reliability and slightly less biased genomic predictions compared with AF from the base population. Although accounting for structure in $\mathbf{G}$ did not yield higher validation reliability, GEBV of some individuals changed markedly. These animals were from the population with a smaller number of animals. Also, coefficients in $\mathbf{G}$ constructed with breed-wise AF from the base population were more comparable with the pedigreebased relationship matrix, which may have optimized the blending of genomic and pedigree information for this admixed population. In the presence of purebreds, 
actual breed proportions derived from marker data may be more beneficial in the estimation of breed-wise AF than expected pedigree-based proportions.

\section{ACKNOWLEDGMENTS}

The authors acknowledge the Nordic Cattle Genetic Evaluation Ltd. (Aarhus, Denmark) and Nordic Genomic Selection project (Aarhus, Denmark) for providing the genotype and phenotype data. M. L. Makgahlela acknowledges financial support from the Finnish Ministry of Agriculture and Forestry (Helsinki, Finland) and the University of Helsinki (Finland).

\section{REFERENCES}

Aguilar, I., I. Misztal, D. L. Johnson, A. Legarra, S. Tsuruta, and T. J. Lawlor. 2010. Hot topic: A unified approach to utilize phenotypic, full pedigree, and genomic information for genetic evaluation of Holstein final score. J. Dairy Sci. 93:743-752.

Brøndum, R. F., E. Rius-Vilarrasa, I. Strandén, G. Su, B. Guldbrandtsen, W. F. Fikse, and M. S. Lund. 2011. Reliabilities of genomic prediction using combined reference data of the Nordic Red dairy cattle populations. J. Dairy Sci. 94:4700-4707.

Calus, M. P. L., T. H. E. Meuwissen, A. P. W. de Roos, and R F. Veerkamp. 2008. Accuracy of genomic selection using different methods to define haplotypes. Genetics 178:553-561.

Chen, C. Y., I. Misztal, I. Aguilar, A. Legarra, and W. M. Muir. 2011. Effect of different genomic relationship matrices on accuracy and scale. J. Anim. Sci. 89:2673-2679.

Christensen, O. F., and M. S. Lund. 2010. Genomic prediction when some animals are not genotyped. Genet. Sel. Evol. 42:2.

Christensen, O. F., P. Madsen, B. Nielsen, T. Ostersen, and G. Su. 2012. Single-step methods for genomic evaluation in pigs. Animal 6:1565-1571.

Daetwyler, H. D., B. Villanueva, and J. A. Woolliams. 2008. Accuracy of predicting the genetic risk of disease using a genome-wide approach. PLoS ONE 3:e3395.

de Roos, A. P. W., B. J. Hayes, and M. E. Goddard. 2009. Reliability of genomic predictions across multiple populations. Genetics 183:1545-1553.

de Roos, A. P. W., C. Schrooten, and T. Druet. 2011. Genomic breeding value estimation using genetic markers, inferred ancestral haplotypes, and the genomic relationship matrix. J. Dairy Sci. 94:4708-4714.

Deng, H. W. 2001. Population admixture may appear to mask, change or reverse genetic effects of genes underlying complex traits. Genetics 159:1319-1323.

Forni, S., I. Aguilar, and I. Misztal. 2011. Different genomic relationship matrices for single-step analysis using phenotypic, pedigree and genomic information. Genet. Sel. Evol. 43:1.

Frkonja, A., B. Gredler, U. Schnyder, I. Curik, and J. Sölkner. 2012. Prediction of breed composition in an admixed cattle population. Anim. Genet. 43:696-703.

Gao, H., O. F. Christensen, P. Madsen, U. S. Nielsen, Y. Zhang, M. S. Lund, and G. Su. 2012. Comparison on genomic predictions using three GBLUP methods and two single-step blending methods in the Nordic Holstein population. Genet. Sel. Evol. 44:8.

Gengler, N., P. Mayeres, and M. Szydlowski. 2007. A simple method to approximate gene content in large pedigree populations: Application to the myostatin gene in dual-purpose Belgian Blue cattle. Animal 1:21-28.

Harris, B. L., F. E. Creagh, A. M. Winkelman, and D. L. Johnson. 2011. Experiences with the Illumina high density bovine BeadChip. Interbull Bull. 44:3-7.
Harris, B. L., and D. L. Johnson. 2010. Genomic predictions for New Zealand dairy bulls and integration with national genetic evaluation. J. Dairy Sci. 93:1243-1252.

Harris, B. L., A. M. Winkelman, and D. L. Johnson. 2012. Large-scale single-step genomic evaluation for milk production traits. Interbull Bull. 46:20-24.

Hayes, B. J., P. J. Bowman, A. C. Chamberlain, K. Verbyla, and M. E. Goddard. 2009a. Accuracy of genomic breeding values in multibreed dairy cattle populations. Genet. Sel. Evol. 41:51.

Hayes, B. J., P. J. Bowman, A. J. Chamberlain, and M. E. Goddard. 2009b. Invited review: Genomic selection in dairy cattle: Progress and challenges. J. Dairy Sci. 92:433-443.

Ibáñez-Escriche, N., R. L. Fernando, A. Toosi, and J. C. M. Dekkers. 2009. Genomic selection of purebreds for crossbred performance. Genet. Sel. Evol. 41:12.

Illumina Inc. 2005. Illumina GenCall Data Analysis Software-GenCall software algorithms for clustering, calling, and scoring genotypes. Illumina Pub. No. 370-2004-009. Illumina Inc., San Diego, CA.

Interbull. 2004. Code of Practice; version April 27, 2004. https://wiki. interbull.org/public/wholeCoP_toPrint?action=print.

Jairath, L., J. C. M. Dekkers, L. R. Schaeffer, Z. Liu, E. B. Burnside, and B. Kolstad. 1998. Genetic evaluation for herd life in Canada. J. Dairy Sci. 81:550-562.

Janss, L., G. de los Campos, N. Sheehan, and D. Sorensen. 2012. Inferences from genomic models in stratified populations. Genetics 192:693-704.

Kearney, F., A. Cromie, and D. P. Berry. 2009. Implementation and uptake of genomic evaluations in Ireland. Interbull Bull. 40:227230.

Koivula, M., I. Strandén, G. Su, and E. A. Mäntysaari. 2012. Different methods to calculate genomic predictions - Comparisons of BLUP at the single nucleotide polymorphism level (SNP-BLUP), BLUP at the individual level (G-BLUP), and the one-step approach (HBLUP). J. Dairy Sci. 95:4065-4073.

Kuehn, L. A., J. W. Keele, G. L. Bennett, T. G. McDaneld, T. P. L. Smith, W. M. Snelling, T. S. Sonstegard, and R. M. Thallman. 2011. Predicting breed composition using breed frequencies of 50,000 markers from the US Meat Animal Research Center 2,000 bull project. J. Anim. Sci. 89:1742-1750.

Lidauer, M., E. A. Mäntysaari, I. Strandén, J. Pösö, J. Pedersen, U. S. Nielsen, K. Johansson, J.-A. Eriksson, P. Madsen, and G. P. Aamand. 2006. Random heterosis and recombination loss effects in a multibreed evaluation for Nordic Red dairy cattle. Commun. No. 24-02 in Proc. 8th World Congr. Genet. Appl. Livest. Prod., Belo Horizonte, Brazil. Instituto Prociência, Minas Gerais, Brazil.

Lidauer, M., and I. Strandén. 1999. Fast and flexible program for genetic evaluation in dairy cattle. Interbull Bull. 20:20.

Makgahlela, M. L., E. A. Mäntysaari, I. Strandén, M. Koivula, U. S. Nielsen, M. J. Sillanpää, and J. Juga. 2013a. Across breed multitrait random regression genomic predictions in the Nordic Red dairy cattle. J. Anim. Breed. Genet. 130:10-19.

Makgahlela, M. L., I. Strandén, U. S. Nielsen, M. J. Sillanpää, and E. A. Mäntysaari. 2013b. The estimation of genomic relationships using breedwise allele frequencies among animals in multibreed populations. J. Dairy Sci. 96:5364-5375.

Mäntysaari, E. A., M. Koivula, I. Strandén, J. Pösö, and G. P. Aamand. 2011. Estimation of GEBV using deregressed individual cow breeding values. Interbull Bull. 44:26-29.

Mäntysaari, E. A., Z. Liu, and P. VanRaden. 2010. Interbull validation test for genomic evaluations. Interbull Bull. 41:17-22.

Meuwissen, T. H. E., B. J. Hayes, and M. E. Goddard. 2001. Prediction of total genetic value using genome-wide dense marker maps. Genetics 157:1819-1829.

Meuwissen, T. H. E., T. Luan, and J. A. Woolliams. 2011. The unified approach to the use of genomic and pedigree information in genomic evaluations revisited. J. Anim. Breed. Genet. 128:429-439.

Misztal, I., A. Legarra, and I. Aguilar. 2009. Computing procedures for genetic evaluation including phenotypic, full pedigree, and genomic information. J. Dairy Sci. 92:4648-4655. 
Olson, K. M., P. M. VanRaden, and M. E. Tooker. 2012. Multibreed genomic evaluations using purebred Holsteins, Jerseys, and Brown Swiss. J. Dairy Sci. 95:5378-5383.

Olson, K. M., P. M. VanRaden, M. E. Tooker, and T. A. Cooper. 2011. Differences among methods to validate genomic evaluations for dairy cattle. J. Dairy Sci. 94:2613-2620.

Price, A. L., N. A. Zaitlen, D. Reich, and N. Patterson. 2010. New approaches to population stratification in genome-wide association studies. Nat. Rev. Genet. 11:459-463.

Pryce, J. E., B. Gredler, S. Bolormaa, P. J. Bowman, C. Egger-Danner, C. Fuerst, R. Emmerling, J. Sölkner, M. E. Goddard, and B. J. Hayes. 2011. Short communication: Genomic selection using a multi-breed, across-country reference population. J. Dairy Sci. 94:2625-2630.

Reinhardt, F., Z. Liu, F. Seefried, and G. Thaller. 2009. Implementation of genomic evaluation in German Holsteins. Interbull Bull. 40:219-226.

Rius-Vilarrasa, E., T. Iso-Touru, I. Strandén, N. Schulman, B. Guldbrandtsen, E. Strandberg, M. S. Lund, J. Vilkki, and W. F. Fikse. 2011. Characterization of linkage disequilibrium in a Danish, Swedish and Finnish Red Breed cattle population. Page 177 in Proc. 62nd Annu. Mtg. Eur. Fed. Anim. Sci., Stavanger, Norway. Wageningen Academic Publishers, Wageningen, the Netherlands.

Schaeffer, L. R. 2001. Multiple trait international bull comparisons. Livest. Prod. Sci. 69:145-153.

Scheet, P., and M. Stephens. 2006. A fast and flexible statistical model for large-scale population genotype data: Applications to inferring missing genotypes and haplotypic phase. Am. J. Hum. Genet. 78:629-644.

Simeone, R., I. Misztal, I. Aguilar, and A. Legarra. 2011. Evaluation of the utility of diagonal elements of the genomic relationship matrix as a diagnostic tool to detect mislabelled genotyped animals in a broiler chicken population. J. Anim. Breed. Genet. 128:386-393.

Strandén, I., and O. F. Christensen. 2011. Allele coding in genomic evaluation. Genet. Sel. Evol. 43:25

Strandén, I., and E. A. Mäntysaari. 2010. A recipe for multiple trait deregression. Interbull Bull. 42:21-24.

Su, G., R. F. Brøndum, P. Ma, B. Guldbrandtsen, G. P. Aamand, and M. S. Lund. 2012a. Comparison of genomic predictions using medium-density $(\sim 54,000)$ and high-density $(\sim 777,000)$ single nucleotide polymorphism marker panels in Nordic Holstein and Red Dairy Cattle populations. J. Dairy Sci. 95:4657-4665.

Su, G., B. Guldbrandtsen, V. R. Gregersen, and M. S. Lund. 2010. Preliminary investigation on reliability of genomic estimated breeding values in the Danish Holstein population. J. Dairy Sci. 93:1175-1183.

Su, G., P. Madsen, U. S. Nielsen, E. A. Mäntysaari, G. P. Aamand, O. F. Christensen, and M. S. Lund. 2012b. Genomic prediction for Nordic Red Cattle using one-step and selection index blending. J. Dairy Sci. 95:909-917.

Sul, J. H., and E. Eskin. 2013. Mixed models can correct for population structure for genomic regions under selection. Nat. Rev. Genet. 14:300.

Sullivan, P. G., and P. M. VanRaden. 2009. Development of genomic GMACE. Interbull Bull. 40:157-161.

Thomasen, J. R., B. Guldbrandtsen, G. Su, R. F. Brondum, and M. S. Lund. 2012. Reliabilities of genomic estimated breeding values in Danish Jersey. Animal 6:789-796.

Van Doormaal, B. J., G. J. Kistemaker, P. G. Sullivan, M. Sargolzaei, and F. S. Schenkel. 2009. Canadian implementation of genomic evaluations. Interbull Bull. 40:214-217.

VanRaden, P. M. 2008. Efficient methods to compute genomic predictions. J. Dairy Sci. 91:4414-4423.

VanRaden, P. M., C. P. Van Tassell, G. R. Wiggans, T. S. Sonstegard, R. D. Schnabel, J. F. Taylor, and F. S. Schenkel. 2009. Invited review: Reliability of genomic predictions for North American Holstein bulls. J. Dairy Sci. 92:16-24.

Vitezica, Z. G., I. Aguilar, I. Misztal, and A. Legarra. 2011. Bias in genomic predictions for populations under selection. Genet. Res. (Camb.) 93:357-366

Yang, J., B. Benyamin, B. P. McEvoy, S. Gordon, A. K. Henders, D R. Nyholt, P. A. Madden, A. C. Heath, N. G. Martin, G. W. Montgomery, M. E. Goddard, and P. M. Visscher. 2010. Common SNPs explain a large proportion of the heritability for human height. Nat. Genet. 42:565-569.

Zimin, A. V., A. L. Delcher, L. Florea, D. R. Kelley, M. C. Schatz, D. Puiu, F. Hanrahan, G. Pertea, C. P. Van Tassell, T. S. Sonstegard, G. Marçais, M. Roberts, P. Subramanian, J. A. Yorke, and S. L. Salzberg. 2009. A whole-genome assembly of the domestic cow, Bos taurus. Genome Biol. 10:R42. 